\title{
Genetic influence on cognitive development between childhood and adulthood
}

\author{
Josephine Mollon ${ }^{1}$ - Emma E. M. Knowles $\mathbb{1}^{1}$ - Samuel R. Mathias ${ }^{1} \cdot$ Ruben Gur $^{2}$ - Juan Manuel Peralta ${ }^{3}$. \\ Daniel J. Weiner ${ }^{4,5,6}$ - Elise B. Robinson ${ }^{4,5,6} \cdot$ Raquel E. Gur $^{2}$ - John Blangero ${ }^{3}$ - Laura Almasy ${ }^{7}$ David C. Glahn $\mathbb{B}^{1,8}$
}

Received: 17 April 2018 / Revised: 15 August 2018 / Accepted: 11 September 2018 / Published online: 19 October 2018

(c) Springer Nature Limited 2018

\begin{abstract}
Successful cognitive development between childhood and adulthood has important consequences for future mental and physical wellbeing, as well as occupational and financial success. Therefore, delineating the genetic influences underlying changes in cognitive abilities during this developmental period will provide important insights into the biological mechanisms that govern both typical and atypical maturation. Using data from the Philadelphia Neurodevelopmental Cohort (PNC), a large population-based sample of individuals aged 8 to 21 years old $(n=6634)$, we used an empirical relatedness matrix to establish the heritability of general and specific cognitive functions and determine if genetic factors influence cognitive maturation (i.e., Gene $\times$ Age interactions) between childhood and early adulthood. We found that neurocognitive measures across childhood and early adulthood were significantly heritable. Moreover, genetic variance on general cognitive ability, or $g$, increased significantly between childhood and early adulthood. Finally, we did not find evidence for decay in genetic correlation on neurocognition throughout childhood and adulthood, suggesting that the same genetic factors underlie cognition at different ages throughout this developmental period. Establishing significant Gene $\times$ Age interactions in neurocognitive functions across childhood and early adulthood is a necessary first step in identifying genes that influence cognitive development, rather than genes that influence cognition per se. Moreover, since aberrant cognitive development confers risk for several psychiatric disorders, further examination of these Gene $\times$ Age interactions may provide important insights into their etiology.
\end{abstract}

Electronic supplementary material The online version of this article (https://doi.org/10.1038/s41380-018-0277-0) contains supplementary material, which is available to authorized users.

$\triangle$ Josephine Mollon

josephine.mollon@yale.edu

1 Department of Psychiatry, Yale University School of Medicine, New Haven, CT, USA

2 Department of Psychiatry, Perelman School of Medicine, and the Penn-CHOP Lifespan Brain Institute, University of Pennsylvania, Philadelphia, PA, USA

3 South Texas Diabetes and Obesity Institute, School of Medicine, University of Texas of the Rio Grande Valley, Brownsville, TX, USA

4 Analytic and Translational Genetics Unit, Department of

\section{Introduction}

The developmental period between childhood and early adulthood is a time of substantial cognitive change, with significant gains in working memory, complex reasoning and social abilities [1,2]. Successful cognitive development during this period has important consequences for future mental and physical wellbeing, as well as occupational and

Medicine, Massachusetts General Hospital and Harvard Medical School, Boston, Massachusetts, USA

5 Stanley Center for Psychiatric Research, Broad Institute of MIT and Harvard, Cambridge, Massachusetts, USA

6 Program in Medical and Population Genetics, Broad Institute of MIT and Harvard, Cambridge, Massachusetts, USA

7 Department of Genetics, Perelman School of Medicine, and the Penn-CHOP Lifespan Brain Institute, University of Pennsylvania, Philadelphia, PA, USA

8 Olin Neuropsychiatry Research Center, Institute of Living, Hartford, CT, USA 
financial success [3]. This period is also thought to be a critical window of risk for many psychiatric illnesses, such as psychotic disorders [4, 5]. While both biological and environmental factors clearly influence cognitive development, most current work in neurodevelopment focuses on very early stages (e.g., prenatal/perinatal; first 12 months) $[6,7]$. Moreover, a rapidly growing literature has documented changes in gene expression over the course of brain development [8-10], but the relationship between temporal variation in gene expression and cognitive development is unclear. Delineating the genetic influences on cognitive development between childhood and adulthood should provide important insights into the biological mechanisms governing both typical and atypical maturation.

Cognitive abilities are substantially influenced by genes, with approximately half of the variance in general cognition attributed to genetic factors [11]. Specific abilities, including attention [12-15], working memory [15-19], and declarative memory [20-22], are also heritable. Furthermore, the heritability of cognition is moderated by age, with the heritability of IQ increasing from around $40 \%$ in early childhood to over $80 \%$ in adulthood [23]. More recently, the heritability of general cognition has been shown to increase between childhood/adolescence and early adulthood [11, 24]. Between early adulthood and old age, on the other hand, heritability of processing speed and memory decreases [25]. Thus, genetic influences on cognition may vary, not as a linear function of age, but depending on the developmental period and the specific cognitive abilities under investigation [26]. While the moderation of heritability by age is well replicated [3], previous studies have been unable to fully disentangle this effect because age has been categorized into broad developmental periods, rather than investigated as a continuous factor. Moreover, heritability at different ages has typically been estimated in different samples.

Early efforts to explain the increasing heritability of cognition across development mostly focused on twin and adoption designs [27-29]. More recently, studies have examined the genetic influence of common variants in unrelated individuals, known as SNP-based heritability [30, 31]. For example, Trzaskowski and colleagues [28] examined changes in heritability of general intelligence, or $g$, between ages 7 and 12 in 2875 unrelated children, as well as 6702 twin pairs. SNP-based heritabilities were 0.26 at age 7 and 0.45 at age 12 , similar to the twin-based heritabilities of 0.36 and 0.49 at these ages. While these studies provide initial insight into the genetic mechanisms underlying cognitive development, they have a number of limitations. First, most have focused on childhood, with only one study investigating the period between childhood and adolescence [32] and none spanning from childhood to adulthood. Second, using different cognitive tests at different ages has introduced variability, making it difficult to establish whether cognitive changes stem from genetic or methodological factors. Finally, most studies have focused on general cognition, and have not applied a consistent genetic approach to specific functions in a single sample [15].

Since increase in age is not the direct consequence of gene action, increase in age, or maturation, can be considered an effect of the environment and modeled as a Gene $\times$ Environment interaction. Thus, a Gene $\times$ Age $(\mathrm{G} \times \mathrm{A})$ interaction on cognitive development can be tested using a cross-sectional design that models differences in cognitive performance as a function of both relatedness (empirically defined) and similarity in age between individuals [25, 33]. As well as providing an estimate of genetic influence on cognitive development, $\mathrm{G} \times \mathrm{A}$ interaction analysis suggests whether this effect is due to fluctuations in action of the same genetic factors, or variation in the genetic factors influencing the trait at different ages. Glahn and colleagues [25] used this approach to identify neurocognitive processes with significant $\mathrm{G} \times \mathrm{A}$ interactions, identifying potential phenotypes for gene discovery in age-related cognitive decline. Similarly, Kent and colleagues [34] identified more than 600 lymphocyte-based RNA transcripts with significant $\mathrm{G} \times \mathrm{A}$ interactions, defining candidate genes for biological aging. Despite the high heritability of cognitive functions, identifying specific genes that influence cognition has proved challenging. Considering $\mathrm{G} \times \mathrm{A}$ interactions in the search for cognition genes could help, particularly when the goal is to determine if the gene influences cognitive development.

In this study, we modeled change in cognitive functions between childhood and early adulthood in the Philadelphia Neurodevelopmental Cohort [35], a large population-based sample of individuals aged 8-21 years old. The aims of the study were to use an empirical relatedness matrix to (1) establish the heritability of general and specific cognitive functions, and (2) determine if $\mathrm{G} \times \mathrm{A}$ interactions influence these functions between childhood and early adulthood.

\section{Methods}

\section{Participants}

The Philadelphia Neurodevelopmental Cohort (PNC) is a population-based sample from the greater Philadelphia area, comprising 9421 individuals aged 8-21 years who received medical care within the Children's Hospital of Philadelphia network. Study procedures have been described in detail elsewhere [35]. Briefly, participants presented for a range of medical needs, including general checkups, and chronic condition management [28]. Participants provided written assent/consent for genomic studies upon providing blood 
samples during the clinical visit. Inclusion criteria were: (1) ability to provide signed informed consent (parental consent was required for participants under age 18), (2) English language proficiency, and (3) physical and cognitive ability to participate in computerized cognitive testing. Data deposited in $\mathrm{dbGaP}[36]$ were used in the present analyses. The present analyses were limited to participants who identified as either white non-Hispanic (European American) or black non-Hispanic (African American). A total of 6634 subjects with available cognitive and genetic data were included in the analyses, of whom 4694 (70.8\%) were European American (EA) and 1940 (29.2\%) were African American (AA). Given known differences in minor allele frequencies between individuals of African and European ancestry, all genetic analyses were conducted separately for EAs and AAs. Age ranged from 8 to 21, with a mean of $13.9(\mathrm{SD}=3.65)$ (Figure $\mathrm{S} 1), 49.1 \%$ of subjects were male $(n=3254)$.

\section{Neurocognitive assessment}

All PNC participants completed the 1-hour Computerized Neurocognitive Battery (CNB) [37, 38]. The CNB consists of 14 tests designed to capture functioning in five domains of cognitive ability: (1) executive function (abstraction and mental flexibility, attention, working memory), (2) episodic memory (verbal, facial, spatial), (3) complex cognition (verbal reasoning, nonverbal reasoning, spatial processing), (4) social cognition (emotion identification, emotion differentiation, age differentiation), and (5) sensorimotor speed (motor, sensorimotor). The CNB has been described elsewhere $[37,38]$ and a summary of the measures is included in Table S1. The battery also included the reading component of the Wide Range Achievement Test (WRAT), a measure of general cognitive ability.

In addition to the measures directly indexed by the $\mathrm{CNB}$, we derived a general composite score $(g)$ as the first component of principal component analyses (PCA) using all tests except the WRAT. We also derived a general composite score for speed $(g s)$ as the first component of PCA using reaction times for all cognitive measures. To minimize the impact of missing data on these composite scores, the Multivariate Imputation by Chained Equation (MICE) method [39-41] was used to impute missing values using the mice package in $\mathrm{R}$ [42]. The imputation model was based on age, sex, and ethnicity (AA or EA). Test scores were imputed for subjects with less than $50 \%$ missing neurocognitive data and five datasets were imputed (see Figure S2 for patterns of missingness and Figure S3 for plots of observed and imputed data). All subsequent analyses were conducted on the imputed neurocognitive data. Correlations among all test scores can be seen in Figure S4.

\section{Genotyping}

Samples were genotyped on one of four Illumina arrays: HumanHap550, HumanHap610, OmniExpress, or Human1M. Genotyped data were imputed in a separate phase of the study at the Broad Institute [43]. Unobserved genotypes from each chip set were imputed using the IMPUTE2 package and the reference haplotypes in Phase I of the 1000 genomes data (June 2011 release) that included 37138905 variants from 1094 individuals from Africa, Asia, Europe, and the Americas. The imputed genotype data were used in subsequent analyses. All analyses were conducted separately for EA and AA populations.

\section{Estimation of the empirical relatedness matrix}

Empirical relatedness quantifies the proportion of alleles that are identical by descent between individuals and was calculated for all pairs of individuals using the genotype data. A set of $50 \mathrm{k}$ common autosomal SNPs in approximate linkage equilibrium was selected from all available SNP variants after LD pruning $\left(r^{2}>0.1\right)$ using PLINK [44]. Relatedness was estimated from the selected SNPs using the IBDLD software package [45] (up to 50 SNPs within a $2 \mathrm{cM}$ span), and a whitening transformation was applied to the resulting empirical relatedness matrix. The matrix was inspected to ensure correct properties (trace equal to number of genotyped subjects, symmetry, positive semi-definiteness, range of diagonal, and off-diagonal elements). The distribution of estimated relatedness values can be seen in Figure S5.

\section{Statistical and quantitative genetic analyses}

The statistical programming language $\mathrm{R}$ [46] was used for descriptive statistics and graphics. All genetic analyses were conducted using the SOLAR software package [47]. Briefly, SOLAR implements linear mixed-effects models, which decompose the overall variance of a quantitative trait. Traditionally, these analyses have been performed on family data using matrices calculated from pedigree information, but can also be applied to cohorts of related and unrelated individuals using relatedness estimated from genotype information [48]. Under a simple polygenic model, the phenotypic variance $\left(\sigma_{p}^{2}\right)$ is assumed to be composed of an additive genetic component $\left(\sigma_{g}^{2}\right)$ and an environmental component $\left(\sigma_{e}^{2}\right)$. Maximum-likelihood estimates (MLEs) of $\sigma_{g}^{2}$ and $\sigma_{e}^{2}$ (along with regression coefficients for any variables included as fixed-effect covariates in the model) are found using an iterative procedure. Narrow-sense heritability $\left(h^{2}\right)$ is the proportion of the phenotypic variance accounted for by additive genetic variance $\left(h^{2}=\sigma_{g}^{2} / \sigma_{p}^{2}\right)$. 


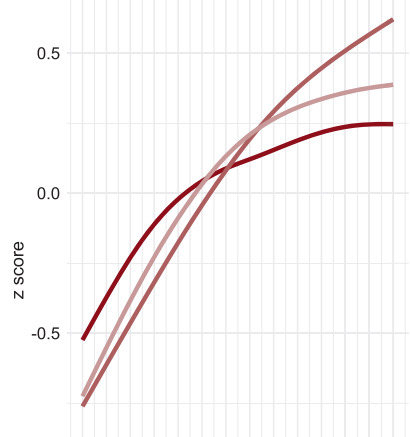

$-1.0$

89101112131415161718192021 Age

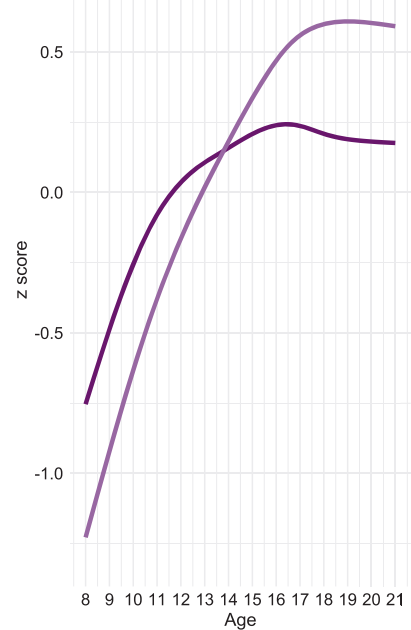

Neurocognitive scores by age for all participants

Fig. 1 Neurocognitive scores by age for all participants

As detailed previously $[25,34,49]$, this polygenic model can be extended to examine Gene $\times$ Environment $(\mathrm{G} \times \mathrm{E})$ interactions. One potential consequence of a $G \times E$ interaction is that the overall additive genetic variance is greater under certain environmental conditions than others. To test for this effect with a quantitatively measured environment, the polygenic model is modified to include a linear function on the logarithm of $\sigma_{g}^{2}$. This linear function contains a free parameter, $\gamma$, reflecting the change in $\sigma_{g}^{2}$ per unit of the environmental variable, age in this case. A non-zero value of $\gamma$ implies a heritable response to the environment, and therefore, a $\mathrm{G} \times \mathrm{E}$ interaction. A second potential consequence of a $\mathrm{G} \times \mathrm{E}$ interaction is that the trait exhibits imperfect pleiotropy with itself at different ages i.e., the relative contributions of genetic factors to $\sigma_{g}^{2}$ change with age [34]. In this case, the genetic correlation $\left(\rho_{g}\right)$ between the trait measured at one age and the same trait measured at another age is less than 1 , suggesting changes in the genetic
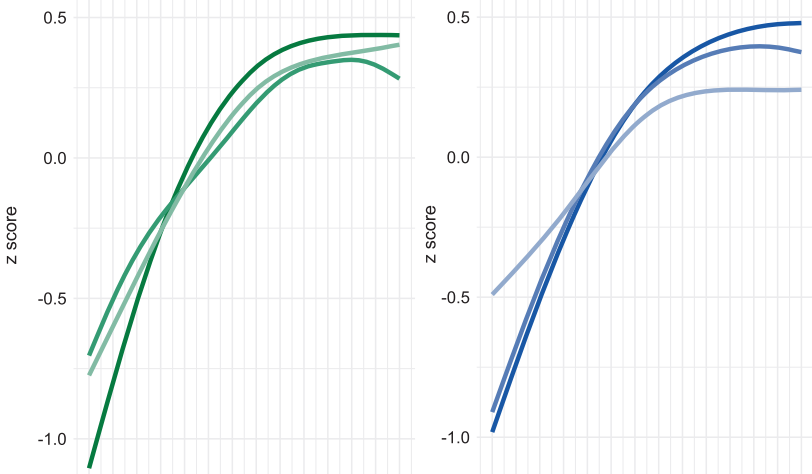

89101112131415161718192021
Age

89101112131415161718192021
Age

Measure

Abstraction

Attention

Working memory

Face memory

Spatial memory

Verbal memory

Verbal reasoning

Nonverbal reasoning

Spatial reasoning

Age differentiation

Age differentiation
Emotion differentiation

Emotion identification

Motor speed

Sensorimotor speed

WRAT

Composite score (g)

Speed composite score (gs)

factors contributing to $\sigma_{g}^{2}$. This phenomenon can be examined in cross-sectional studies where individuals are only tested under a single environmental condition or at a single time point, provided the degree of relatedness between individuals is known [33]. To uncover this effect, the $\rho_{g}$ for a given pair of individuals is modeled as a function of the difference in their ages and another free parameter, $\lambda$, reflecting the rate of decay in $\rho_{g}$ as the difference in ages increases. The genetic correlation $\left(\rho_{g}\right)$ equals 1 if either the difference in ages between individuals is 0 or $\lambda$ is 0 . Thus, a non-zero value of $\lambda$ implies decreasing $\rho_{g}$ and imperfect pleiotropy across ages, and therefore, a $\mathrm{G} \times \mathrm{E}$ interaction. See Genotype $\times$ Age Interaction Model in supplement for more information.

Polygenic models with modifications to test for both consequences of a potential $\mathrm{G} \times \mathrm{E}$ interaction, i.e., $\gamma$ and $\lambda$, were fitted to all neurocognitive traits. Age in years was fitted as the continuous environmental variable. All models 
included age, age ${ }^{2}$, sex, and their interactions as fixed-effect covariates. Statistical significance for each of the parameters of interest was determined by comparing the likelihood of the full polygenic model to the likelihood of a null model i.e., where the parameter of interest was constrained to 0 . To control for multiple testing, the false discovery rate (FDR) was set at 5\% [50]. A rank-based inverse normal transformation was applied to scores on each test to ensure normal distributions. Separate polygenic models were fitted to data from EAs and AAs.

\section{Results}

\section{Cognitive scores increase between childhood and early adulthood}

Figure 1 shows neurocognitive test scores plotted by age. As previously reported [1], increasing age was significantly associated with increasing test scores across all neurocognitive measures. Verbal reasoning, age differentiation, sensorimotor speed, WRAT, and $g$ showed particularly substantial age-related changes, with increases of 1.69, 1.56, 2.08, 2.08, and 2.08 SD between ages 8 and 21, respectively (Fig. 1).

\section{Cognitive abilities are heritable}

Heritability estimates for all neurocognitive measures are presented in Fig. 2 and Table 1. For EAs, all heritability estimates, except for abstraction and age differentiation, were significant after adjustment for multiple testing (Table 1). For AAs, all heritability estimates, except for abstraction, spatial memory, verbal memory, spatial reasoning, age differentiation, and sensorimotor speed were significant after adjustment for multiple testing (Table 1). The AA sample is smaller than the EA sample, which may account for fewer statistically significant results. Neurocognitive measures with non-significant heritability estimates were excluded from subsequent $\mathrm{G} \times \mathrm{A}$ analyses. Figure S6 shows heritability estimates for the imputed and unimputed neurocognitive data.

\section{$\mathbf{G} \times \mathbf{A}$ interaction I: genetic variance increases with age}

In EAs, general cognitive ability, or $g$, exhibited a significant increase in genetic variance with increasing age ( $\gamma=0.047, p=0.024)$ after adjustment for multiple testing. This effect is denoted by $\gamma$ and suggests that specific genetic factors influence change in performance on these measures, but also that the magnitude of effect of these genetic factors varies as a function of age (Table 1). Figure 3 shows genetic variance, environmental variance, and heritability between ages 8 and 21 for $g$.

In AAs, increase in genetic variance with increasing age on $g$ showed a trend towards statistical significance after adjustment for multiple testing $(\gamma=0.081, p=0.065)$ (Table 1). Figure S7 shows genetic variance, environmental variance, and heritability between ages 8 and 21 for $g$ in the AA sample. The smaller AA sample may account for fewer statistically significant results.

\section{$\mathbf{G} \times \mathbf{A}$ interaction II: genetic factors influencing cognitive abilities at different ages overlap}

In both EAs and AAs, none of the neurocognitive measures exhibited statistically significant changes in genetic correlation with increasing age, suggesting that the genetic factors influencing changes in neurocognition do not change between childhood and adulthood (Table 1).

\section{Discussion}

Using a large population-based developmental cohort of individuals aged 8-21 years old, we established that both general and specific neurocognitive measures are heritable. Heritability estimates for measures of general cognition, executive function, memory, complex reasoning, social cognition, and sensorimotor speed were moderate to large, consistent with previous findings that neurocognition across the first two decades of life is under considerable genetic influence [11, 24, 32]. Using $\mathrm{G} \times \mathrm{A}$ interaction analyses, we found that specific genetic factors influenced changes in general cognitive ability, or $g$, between childhood and adulthood, but that the scale, or strength, of action of these genetic factors varied with age, particularly in the EA sample. Finally, we did not find evidence for decay in genetic correlation on any neurocognitive measure throughout childhood and early adulthood, suggesting that the same genetic factors influence changes in neurocognition during this developmental period.

Our findings advance knowledge regarding the genetic architecture of cognitive development between childhood and adulthood in several ways. First, while the importance of genetic factors in determining individual differences in general cognitive ability is well established [3], specific neurocognitive functions have received less attention. We found that measures of general cognition, executive function, memory, complex reasoning, social cognition, and sensorimotor speed were significantly heritable. The largest heritability estimates were for general cognitive measures, 67 and $72 \%$ for the WRAT and $g$ respectively, but were also substantial for memory (36-56\%) and complex reasoning (35-46\%) measures. This pattern of results is consistent 
Fig. 2 Heritability estimates for all neurocognitive measures
Population

European American

african American

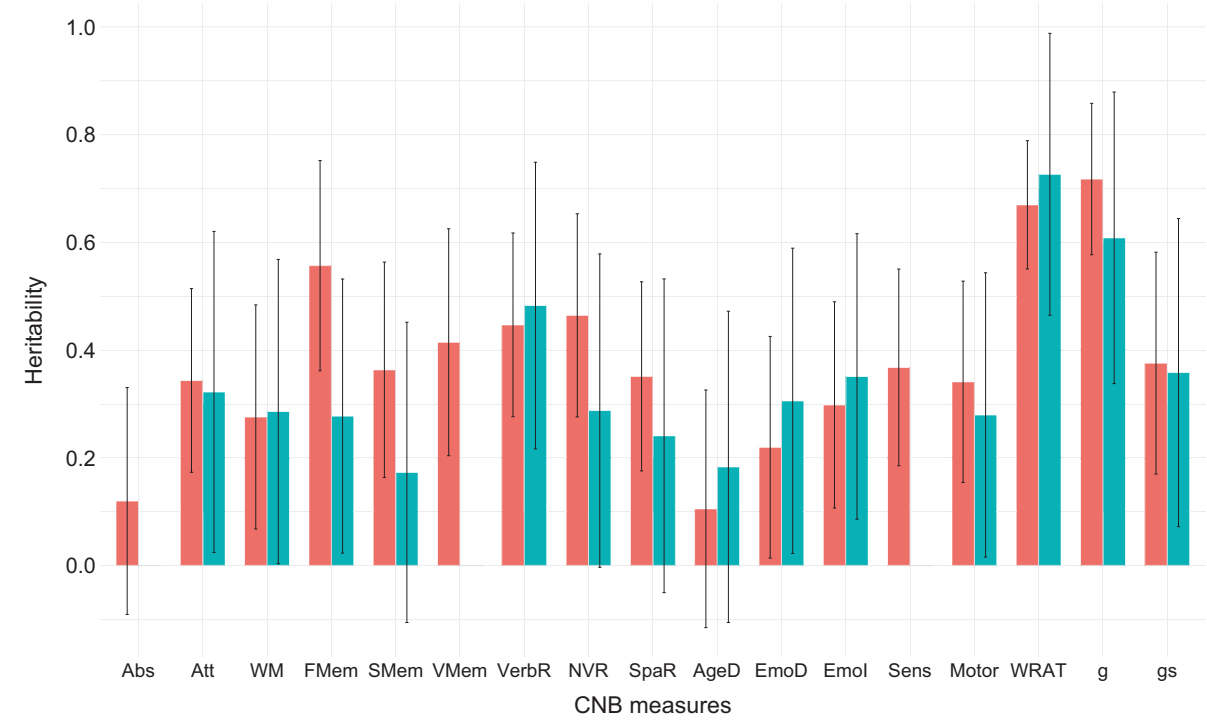

Heritability estimates for all neurocognitive measures

$\mathrm{Abs}=$ Abstraction $/$ mental flexibility; $\mathrm{Att}=$ Attention; $\mathrm{WM}=$ Working memory; $\mathrm{FMem}=$ Face memory; $\mathrm{SMem}=$ Spatial memory; VMem = Verbal memory; VerbR = Verbal Reasoning; NVR = Nonverbal reasoning; SpaR = Spatial reasoning; $\mathrm{AgeD}=$ Age differentiation; $\mathrm{EmoD}=$ Emotion Differentiation; $\mathrm{Emol}=$ Emotion Identification; Sens = Sensorimotor speed; Motor $=$ Motor speed; WRAT $=$ Wide Range Achievement Test (reading); $g$ = Composite score; gs $=$ Speed composite score. Error bars represent $95 \%$ confidence intervals.

with those of genome-wide complex trait analysis (GCTA) in the same sample [15]. Specifically, the largest heritability estimates in both our study and that of Robinson and colleagues are in the complex reasoning domain and the WTAR and the smallest are in the executive and social domains. The main reason for the somewhat higher heritability estimates in our study is that our analyses included all individuals, whereas individuals with relatedness $>0.05$ were excluded prior to GCTA [15]. Thus, the higher heritability estimates using related individuals are likely due to rare variants, $\mathrm{CNVs}$, and structural variants that are not well captured by current common SNPs. Our findings are also in line with reports that heritability increases with increasing task complexity [51-53]. Differences in heritability estimates between cognitive measures may be due to differences in genetic architecture, such that different genetic factors and/or the same genetic factors, but to differing degrees, underlie variation in different neurocognitive measures [54]. Differential effects of environmental factors, such as education [55] and socioeconomic factors [56, 57] may also play a role. Measurement error is unlikely to underlie the observed differences in heritability estimates since reliability of the CNB measures is high ( $>0.7$ for most measures) [15, 38, 58].

Second, $\mathrm{G} \times \mathrm{A}$ analyses revealed a significant age-related increase in genetic variance on general cognitive ability, or $g$, in line with previous studies $[11,28]$. Specifically, we found an increase in genetic variance from 0.32 at age 8 to 0.58 at age 21 , which closely resembles that reported by Trzaskowski and colleagues of an increase from 0.26 to 0.45 between ages 7 and 12 [28]. Similar increases have also been reported in the twin literature. For example, Haworth et al. reported an increase from 0.41 to 0.66 between ages 9 and 17 [11], and Bergen et al. reported an increase from $\sim 0.45-0.80$ between ages 5 and 35 [24]. Previous studies have generally discussed increasing genetic variance in terms of three phenomena: (1) innovation, i.e., novel genetic factors coming into play over time, (2) decay, i.e., existing genetic factors becoming decreasingly important over time, and (3) amplification i.e. existing genetic factors becoming increasingly important over time [27, 29]. Our findings provide evidence for genetic influences on $g$ becoming amplified over childhood and early adulthood. This amplification of genetic factors may be due to gene-environment correlations, whereby individuals increasingly select experiences based on their underlying genetic predispositions, thus accentuating genetic differences [59, 60]. Moreover, genes may become increasingly expressed over the course of cognitive development due to maturational processes [61], and/or environmental factors may moderate gene expression as individuals increasingly select and evoke these factors [11, 62]. Our findings also highlight the importance of $g$, or general cognitive ability, and lend support to the notion of generalist genes i.e., that a 
Table 1 Heritability estimates and $\mathrm{G} \times \mathrm{A}$ interactions

\begin{tabular}{|c|c|c|c|c|c|c|c|c|c|}
\hline Population & Domain & Heritability $\mathrm{h}^{2}$ & $p$ & $\begin{array}{l}\sigma_{\mathrm{g}}^{2} \text { with age } \\
\gamma\end{array}$ & $p$ & $\begin{array}{l}\rho_{\mathrm{g}} \text { with age } \\
\lambda\end{array}$ & $p$ & $\begin{array}{l}\sigma_{\mathrm{e}}^{2} \text { with } \\
\text { age }\end{array}$ & $p$ \\
\hline \multirow{17}{*}{$\begin{array}{l}\text { European Americans } \\
(n=4694)\end{array}$} & Abstraction & 0.12 & 0.135 & - & - & - & - & - & - \\
\hline & Attention & 0.34 & $2 \times 10^{-5}$ & 0.002 & 0.955 & 0.050 & 0.5 & -0.013 & 0.791 \\
\hline & Working memory & 0.28 & 0.004 & -0.036 & 0.665 & 0.099 & 0.5 & -0.029 & 0.692 \\
\hline & Face memory & 0.56 & $6 \times 10^{-7}$ & 0.038 & 0.287 & 0.000 & 0.5 & 0.029 & 0.718 \\
\hline & Spatial memory & 0.36 & $3 \times 10^{-4}$ & 0.124 & 0.100 & 0.000 & 0.5 & -0.056 & 0.318 \\
\hline & Verbal memory & 0.42 & $2 \times 10^{-4}$ & -0.035 & 0.713 & 0.112 & 0.5 & 0.041 & 0.791 \\
\hline & Verbal reasoning & 0.45 & $2 \times 10^{-6}$ & -0.001 & 0.955 & 0.000 & 0.5 & -0.007 & 0.848 \\
\hline & Nonverbal reasoning & 0.46 & $5 \times 10^{-6}$ & 0.021 & 0.665 & 0.126 & 0.5 & 0.007 & 0.848 \\
\hline & Spatial reasoning & 0.35 & $1 \times 10^{-4}$ & -0.009 & 0.834 & 0.128 & 0.5 & 0.005 & 0.848 \\
\hline & Age differentiation & 0.11 & 0.176 & - & - & - & - & - & - \\
\hline & Emotion differentiation & 0.22 & 0.017 & -0.043 & 0.713 & 0.242 & 0.5 & 0.033 & 0.791 \\
\hline & Emotion identification & 0.30 & 0.001 & -0.037 & 0.713 & 0.000 & 0.5 & 0.025 & 0.745 \\
\hline & Sensorimotor speed & 0.37 & $6 \times 10^{-5}$ & 0.053 & 0.382 & 0.000 & 0.5 & 0.036 & 0.676 \\
\hline & Motor speed & 0.34 & $3 \times 10^{-4}$ & 0.082 & 0.287 & 0.033 & 0.5 & 0.030 & 0.692 \\
\hline & WRAT & 0.67 & $8 \times 10^{-22}$ & 0.040 & 0.382 & 0.000 & 0.5 & 0.034 & 0.791 \\
\hline & Composite score (g) & 0.72 & $9 \times 10^{-15}$ & 0.047 & 0.024 & 0.000 & 0.5 & 0.046 & 0.692 \\
\hline & $\begin{array}{l}\text { Speed composite score } \\
\text { (gs) }\end{array}$ & 0.38 & $3 \times 10^{-4}$ & 0.041 & 0.638 & 0.182 & 0.5 & -0.016 & 0.848 \\
\hline \multirow{17}{*}{$\begin{array}{l}\text { African Americans } \\
(n=1940)\end{array}$} & Abstraction & 0.00 & 0.5 & - & - & - & - & - & - \\
\hline & Attention & 0.32 & 0.045 & 0.138 & 0.125 & 0.000 & 0.5 & -0.069 & 0.225 \\
\hline & Working memory & 0.29 & 0.045 & 0.085 & 0.409 & 0.000 & 0.5 & -0.067 & 0.225 \\
\hline & Face memory & 0.28 & 0.045 & 0.002 & 0.969 & 0.061 & 0.5 & 0.013 & 0.699 \\
\hline & Spatial memory & 0.17 & 0.138 & - & - & - & - & - & - \\
\hline & Verbal memory & 0.00 & 0.5 & - & - & - & - & - & - \\
\hline & Verbal reasoning & 0.48 & 0.002 & 0.075 & 0.409 & 0.000 & 0.5 & -0.018 & 0.699 \\
\hline & Nonverbal reasoning & 0.29 & 0.045 & 0.013 & 0.933 & 0.000 & 0.5 & 0.051 & 0.286 \\
\hline & Spatial reasoning & 0.24 & 0.075 & - & - & - & - & - & - \\
\hline & Age differentiation & 0.18 & 0.138 & - & - & - & - & - & - \\
\hline & Emotion differentiation & 0.31 & 0.045 & 0.036 & 0.668 & 0.000 & 0.5 & 0.019 & 0.699 \\
\hline & Emotion identification & 0.35 & 0.028 & -0.066 & 0.409 & 0.034 & 0.5 & 0.033 & 0.528 \\
\hline & Sensorimotor speed & 0.00 & 0.5 & - & - & - & - & - & - \\
\hline & Motor speed & 0.28 & 0.045 & -0.075 & 0.668 & 0.000 & 0.5 & 0.074 & 0.528 \\
\hline & WRAT & 0.73 & $4 \times 10^{-6}$ & 0.040 & 0.409 & 0.000 & 0.5 & 0.031 & 0.699 \\
\hline & Composite score (g) & 0.61 & $1 \times 10^{-4}$ & 0.081 & 0.065 & 0.000 & 0.5 & 0.074 & 0.225 \\
\hline & $\begin{array}{l}\text { Speed composite score } \\
\text { (gs) }\end{array}$ & 0.36 & 0.031 & 0.071 & 0.668 & 0.000 & 0.5 & -0.037 & 0.699 \\
\hline
\end{tabular}

Bolded estimates significant after correction for multiple testing $(\mathrm{FDR}=0.05)$

WRAT wide range achievement test

set of generalist genes influence multiple cognitive domains [63]. The generalist gene hypothesis has important practical implications since $g$ can readily be calculated in any study that includes five or more cognitive tests, allowing combining of data from multiple studies and thus increasing power to detect genetic variants underlying cognition [26].

Third, our $\mathrm{G} \times \mathrm{A}$ analyses suggest overlap between the genetic factors influencing changes in neurocognition in childhood and adulthood, since none of the neurocognitive measures exhibited significant changes in genetic correlation with age. This finding is directly in line with that of a previous meta-analytic study, which found that genetic innovation, whereby novel genetic factors come into play over time, ceased after age 8 [29]. Our findings are also in line with an expansive literature documenting stable genetic effects on cognition throughout the lifespan [3]. For example, in a longitudinal twin study, the genetic correlations of full-scale, verbal and performance IQ between 


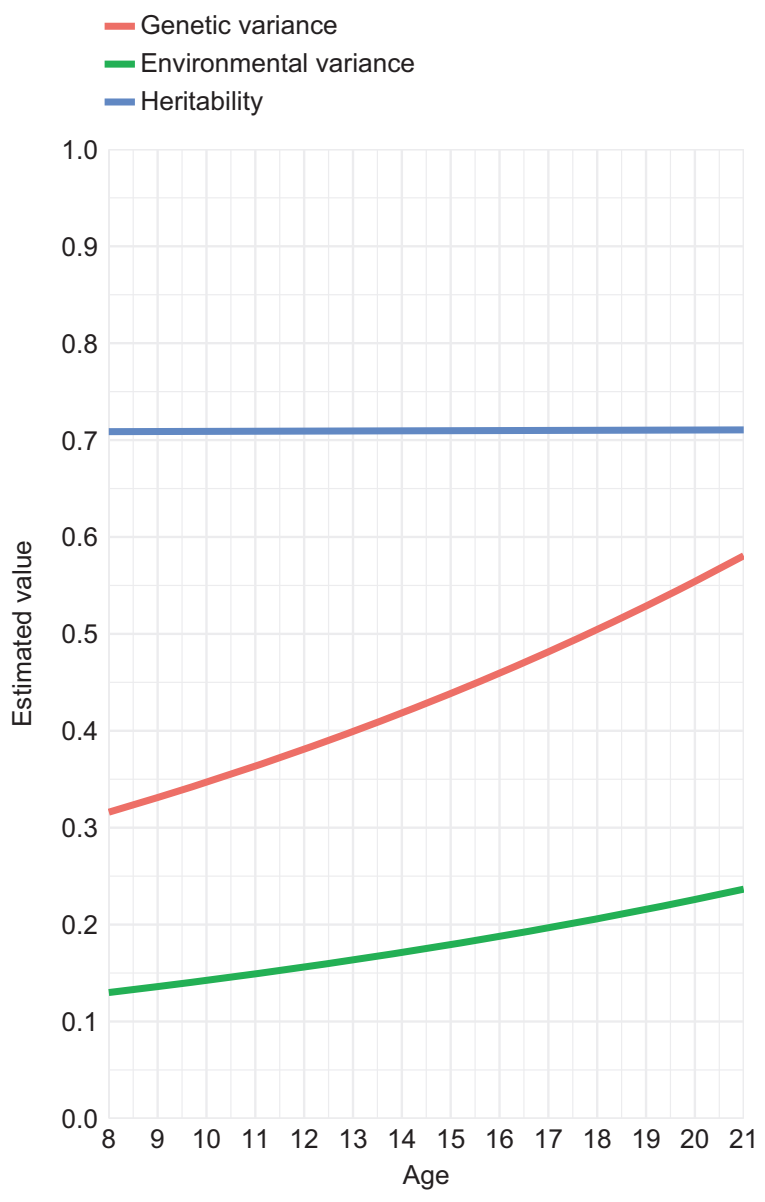

Predicted changes in genetic variance, environmental variance, and heritability with age on general cognitive ability, or $\mathrm{g}$, in the European American group

Fig. 3 Predicted changes in genetic variance, environmental variance, and heritability with age on general cognitive ability, or $g$, in the European American group

childhood and adolescence were estimated at 0.96, 0.78, and 0.90 , respectively [64]. Similarly, a study examining changes in heritability of $g$ between ages 7 and 12 in 6702 twin pairs, as well as 2875 unrelated children [28], reported genetic correlations between ages of 0.73 and 0.75 for the SNP-based and twin approaches, respectively. Our results extend these findings by showing overlap between the genetic factors influencing neurocognition beyond adolescence to early adulthood.

Our findings have several implications and generate testable hypotheses for future work. First, our findings suggest the importance of considering $\mathrm{G} \times \mathrm{A}$ interactions when conducting gene discovery studies since genetic variance increases with age. Thus, gene discovery efforts during this developmental period may be most fruitful when examining young adults or by using statistical models that account for changes in genetic variance with age. Second, this study suggests the feasibility of utilizing different populations of individuals together in genetic studies. Although our AA sample was small, we found significant heritability estimates. Conducting genetic studies across populations is critical in assessing the accuracy and broader relevance of a finding $[65,66]$. Finally, our findings provide evidence for specific, but also dynamic, genetic influences on cognitive development between childhood and adulthood, in line with a growing literature on changes in gene expression over the course of brain development $[8,53]$. Future studies integrating neurocognitive and neurobiological measures will aid understanding of the complex interplay between genetic influences. For example, cortical thickness and white-matter tract integrity may act as intermediary pathways between genetic factors and neurocognition over development [27].

This study has some limitations. First, our data were cross-sectional and longitudinal studies with repeated assessments of the same individuals over time using identical cognitive tests are needed to fully establish developmental cognitive trajectories. Second, while we included individuals of both European and African ancestry, the AA sample was small. Future studies should include larger samples of individuals of African ancestry, as well as other underrepresented populations $[65,66]$. Third, while this is the first time, to our knowledge, that $\mathrm{G} \times \mathrm{A}$ analyses have been used to examine genetic influences on changes in both general and specific cognitive measures between childhood and adulthood, different tests have different psychometric properties and strong assertions about specific cognitive functions require replication in independent samples.

In conclusion, we found that neurocognitive measures across childhood and early adulthood are under substantial genetic influence. Moreover, specific genetic factors influence changes in general cognitive ability, or $g$, between childhood and adulthood, but the magnitude of effect of these genetic factors varies as a function of age. Finally, the genetic factors influencing neurocognition throughout this developmental period overlap at different ages. Establishing the nature of $\mathrm{G} \times \mathrm{A}$ interactions on changes in neurocognition across childhood and early adulthood is a necessary first step in identifying genes that influence cognitive development.

Acknowledgements This research was supported by National Institute of Mental Health grants R01 MH107248 and MH107235.

\section{Compliance with ethical standards}

Conflict of interest The authors declare that they have no conflict of interest.

\section{References}

1. Gur RC, Calkins ME, Satterthwaite TD, Ruparel K, Bilker WB, Moore TM, et al. Neurocognitive growth charting in psychosis spectrum youths. JAMA Psychiatry. 2014;71:366-74. 
2. Paus T. Mapping brain maturation and cognitive development during adolescence. Trends Cogn Sci. 2005;9:60-8.

3. Deary IJ. Intelligence. Annu Rev Psychol. 2012;63:453-82.

4. Reichenberg A, Caspi A, Harrington H, Houts R, Keefe RS, Murray RM, et al. Static and dynamic cognitive deficits in childhood preceding adult schizophrenia: a 30-year study. Am J Psychiatry. 2010;167:160-9.

5. Mollon J, David AS, Zammit S, Lewis G, Reichenberg A. Course of cognitive development from infancy to early adulthood in the psychosis spectrum. JAMA Psychiatry. 2018;75:270-9.

6. Rakic P. Specification of cerebral cortical areas. Science. 1988; 241:170.

7. Rakic P. Evolution of the neocortex: a perspective from developmental biology. Nat Rev Neurosci. 2009;10:724-35.

8. Kang HJ, Kawasawa YI, Cheng F, Zhu Y, Xu X, Li M, et al. Spatio-temporal transcriptome of the human brain. Nature. 2011;478:483-9.

9. Voineagu I, Wang X, Johnston P, Lowe JK, Tian Y, Horvath S, et al. Transcriptomic analysis of autistic brain reveals convergent molecular pathology. Nature. 2011;474:380.

10. Johnson MB, Kawasawa YI, Mason CE, Krsnik Ž, Coppola G, Bogdanović D, et al. Functional and evolutionary insights into human brain development through global transcriptome analysis. Neuron. 2009;62:494-509.

11. Haworth CM, Wright MJ, Luciano M, Martin NG, de Geus EJ, van Beijsterveldt CE, et al. The heritability of general cognitive ability increases linearly from childhood to young adulthood. Mol Psychiatry. 2010;15:1112-20.

12. Fan J, Wu Y, Fossella JA, Posner MI. Assessing the heritability of attentional networks. BMC Neurosci. 2001;2:14.

13. Cornblatt BA, Risch NJ, Faris G, Friedman D, ErlenmeyerKimling L. The Continuous Performance Test, identical pairs version (CPT-IP): I. New findings about sustained attention in normal families. Psychiatry Res. 1988;26:223-38.

14. Myles-Worsley M, Coon H. Genetic and developmental factors in spontaneous selective attention: a study of normal twins. Psychiatry Res. 1997;71:163-74.

15. Robinson EB, Kirby A, Ruparel K, Yang J, McGrath L, Anttila V, et al. The genetic architecture of pediatric cognitive abilities in the Philadelphia Neurodevelopmental Cohort. Mol Psychiatry. 2015; 20:454-8.

16. Ando J, Ono Y, Wright MJ. Genetic structure of spatial and verbal working memory. Behav Genet. 2001;31:615-24.

17. Kremen WS, Jacobsen KC, Xian H, Eisen SA, Eaves LJ, Tsuang MT, et al. Genetics of verbal working memory processes: a twin study of middle-aged men. Neuropsychology. 2007;21:569.

18. Vogler C, Gschwind L, Coynel D, Freytag V, Milnik A, Egli T, et al. Substantial SNP-based heritability estimates for working memory performance. Transl Psychiatry. 2014;4:e438.

19. Knowles EE, Mathias SR, McKay DR, Sprooten E, Blangero J, Almasy L, et al. Genome-wide analyses of working-memory ability: a review. Curr Behav Neurosci Rep. 2014;1:224-33.

20. Bearden CE, Karlsgodt KH, Bachman P, van Erp TG, Winkler AM, Glahn DC. Genetic architecture of declarative memory: implications for complex illnesses. Neuroscientist. 2012;18:516-32.

21. Knowles EE, Carless MA, de Almeida MA, Curran JE, McKay DR, Sprooten E, et al. Genome-wide significant localization for working and spatial memory: Identifying genes for psychosis using models of cognition. Am J Med Genet Part B Neuropsychiatr Genet. 2014;165:84-95.

22. Swan GE, Reed T, Jack LM, Miller BL, Markee T, Wolf PA, et al. Differential genetic influence for components of memory in aging adult twins. Arch Neurol. 1999;56:1127-32.

23. McGue M, Bouchard TJ Jr, Iacono WG, Lykken DT. Behavioral genetics of cognitive ability: A life-span perspective. (eds Plomin R,
McClearn GE), In Nature, nurture \& psychology pp. 59-76 (Washington, DC, US: American Psychological Association, 1993).

24. Bergen SE, Gardner CO, Kendler KS. Age-related changes in heritability of behavioral phenotypes over adolescence and young adulthood: a meta-analysis. Twin Res Hum Genet. 2007; 10:423-33.

25. Glahn DC, Kent JW Jr, Sprooten E, Diego VP, Winkler AM, Curran JE, et al. Genetic basis of neurocognitive decline and reduced white-matter integrity in normal human brain aging. Proc Natl Acad Sci USA. 2013;110:19006-11.

26. Bearden CE, Glahn DC. Cognitive Genomics: searching for the genetic roots of neuropsychological functioning. Neuropsychology. 2018;31:1003-19.

27. Tucker-Drob EM, Briley DA. Continuity of genetic and environmental influences on cognition across the life span: a metaanalysis of longitudinal twin and adoption studies. Psyc. Bull.2014;140:949

28. Trzaskowski M, Yang J, Visscher PM, Plomin R. DNA evidence for strong genetic stability and increasing heritability of intelligence from age 7 to 12 . Mol Psychiatry. 2014;19:380-4.

29. Briley DA, Tucker-Drob EM. Explaining the increasing heritability of cognitive ability across development: a meta-analysis of longitudinal twin and adoption studies. Psychol Sci. 2013;24: 1704-13.

30. Yang J, Lee SH, Goddard ME, Visscher PM. GCTA: a tool for genome-wide complex trait analysis. Am J Hum Genet. 2011; 88:76-82.

31. Yang J, Benyamin B, McEvoy BP, Gordon S, Henders AK, Nyholt DR, et al. Common SNPs explain a large proportion of the heritability for human height. Nat Genet. 2010;42:565-9.

32. Petrill SA, Lipton PA, Hewitt JK, Plomin R, Cherny SS, Corley $\mathrm{R}$, et al. Genetic and environmental contributions to general cognitive ability through the first 16 years of life. Dev Psychol. 2004:40:805-12.

33. Blangero J. Statistical genetic approaches to human adaptability. Hum Biol. 1993;65:5.

34. Kent JW Jr, Goring HH, Charlesworth JC, Drigalenko E, Diego VP, Curran JE, et al. Genotypexage interaction in human transcriptional ageing. Mech Ageing Dev. 2012;133:581-90.

35. Calkins ME, Merikangas KR, Moore TM, Burstein M, Behr MA, Satterthwaite TD, et al. The Philadelphia Neurodevelopmental Cohort: constructing a deep phenotyping collaborative. J Child Psychol Psychiatry. 2015;56:1356-69.

36. Mailman MD, Feolo M, Jin Y, Kimura M, Tryka K, Bagoutdinov $\mathrm{R}$, et al. The NCBI dbGaP database of genotypes and phenotypes. Nat Genet. 2007;39:1181-6.

37. Gur RC, Richard J, Calkins ME, Chiavacci R, Hansen JA, Bilker $\mathrm{WB}$, et al. Age group and sex differences in performance on a computerized neurocognitive battery in children age 8-21. Neuropsychology. 2012;26:251-65.

38. Gur RC, Richard J, Hughett P, Calkins ME, Macy L, Bilker WB, et al. A cognitive neuroscience-based computerized battery for efficient measurement of individual differences: standardization and initial construct validation. J Neurosci Methods. 2010;187:254-62.

39. White IR, Royston P, Wood AM. Multiple imputation using chained equations: issues and guidance for practice. Stat Med. 2011:30:377-99.

40. Rubin DB. Multiple imputation after $18+$ years. J Am Stat Assoc. 1996;91:473-89.

41. Sterne JA, White IR, Carlin JB, Spratt M, Royston P, Kenward MG, et al. Multiple imputation for missing data in epidemiological and clinical research: potential and pitfalls. BMJ. 2009;338: b2393.

42. Buuren Sv, Groothuis-Oudshoorn K mice: Multivariate imputation by chained equations in R. J Stat Soft 2010;45:1-68. 
43. Germine L, Robinson E, Smoller J, Calkins M, Moore T, Hakonarson $\mathrm{H}$, et al. Association between polygenic risk for schizophrenia, neurocognition and social cognition across development. Transl Psychiatry. 2016;6:e924.

44. Chang CC, Chow CC, Tellier LC, Vattikuti S, Purcell SM, Lee JJ. Second-generation PLINK: rising to the challenge of larger and richer datasets. Gigascience. 2015;4:7.

45. Han L, Abney M. Using identity by descent estimation with dense genotype data to detect positive selection. Eur J Hum Genet. 2013;21:205-11.

46. R Development Core Team. R: A language and environment for statistical computing. (Vienna, Austria: R foundation for statistical computing; 2008).

47. Blangero J, Goring HH, Kent JW Jr., Williams JT, Peterson CP, Almasy, et al. Quantitative trait nucleotide analysis using Bayesian model selection. Hum Biol. 2005;77:541-59.

48. Speed D, Hemani G, Johnson MR, Balding DJ. Improved heritability estimation from genome-wide SNPs. Am J Hum Genet. 2012;91:1011-21.

49. Almasy L, Towne B, Peterson C, Blangero J Detecting genotypexage interaction. Genetic Epidemiology 2001;21(S1):S819-24.

50. Benjamini Y, Yekutieli D The control of the false discovery rate in multiple testing under dependency. Ann Stat 2001;29:1165-88.

51. Goldberg Hermo X, Lemos Giraldez S, Fananas Saura L. A systematic review of the complex organization of human cognitive domains and their heritability. Psicothema. 2014;26:1-9.

52. Beaujean AA. Heritability of cognitive abilities as measured by mental chronometric tasks: a meta-analysis. Intelligence. 2005;33: 187-201.

53. Lenroot RK, Giedd JN. The changing impact of genes and environment on brain development during childhood and adolescence: initial findings from a neuroimaging study of pediatric twins. Dev Psychopathol. 2008;20:1161-75.

54. Blokland GAM, Mesholam-Gately RI, Toulopoulou T, Del Re EC, Lam M, DeLisi LE, et al. Heritability of neuropsychological measures in schizophrenia and nonpsychiatric populations: a systematic review and meta-analysis. Schizophr Bull. 2017;43:788-800.
55. Kendler KS, Turkheimer E, Ohlsson H, Sundquist J, Sundquist K. Family environment and the malleability of cognitive ability: a Swedish national home-reared and adopted-away cosibling control study. Proc Natl Acad Sci USA. 2015;112:4612-7.

56. Tucker-Drob EM, Rhemtulla M, Harden KP, Turkheimer E, Fask D. Emergence of a Gene $\mathrm{x}$ socioeconomic status interaction on infant mental ability between 10 months and 2 years. Psychol Sci. 2011;22:125-33.

57. Turkheimer E, Haley A, Waldron M, d'Onofrio B, Gottesman II. Socioeconomic status modifies heritability of IQ in young children. Psychol Sci. 2003;14:623-8.

58. Gur RC, Ragland JD, Moberg PJ, Turner TH, Bilker WB, Kohler $\mathrm{C}$, et al. Computerized neurocognitive scanning: i. methodology and validation in healthy people. Neuropsychopharmacology. 2001;25:766-76.

59. Plomin R, DeFries JC, Loehlin JC. Genotype-environment interaction and correlation in the analysis of human behavior. Psychol Bull. 1977;84:309.

60. Kendler KS, Eaves LJ. Models for the joint effect of genotype and environment on liability to psychiatric illness. Am J Psychiatry. 1986;143:279-89.

61. Cheung AK, Harden KP, Tucker-Drob EM. From specialist to generalist: developmental transformations in the genetic structure of early child abilities. Dev Psychobiol. 2015;57:566-83.

62. Plomin R. Genetics and general cognitive ability. Nature. 1999;402 (6761supp):C25.

63. Kovas Y, Voronin I, Kaydalov A, Malykh SB, Dale PS, Plomin R. Literacy and numeracy are more heritable than intelligence in primary school. Psychol Sci. 2013;24:2048-56.

64. Van Soelen IL, Brouwer RM, Van Leeuwen M, Kahn RS, Pol $\mathrm{HEH}$, Boomsma DI. Heritability of verbal and performance intelligence in a pediatric longitudinal sample. Twin Res Human Genet. 2011;14:119-28.

65. Need AC, Goldstein DB. Next generation disparities in human genomics: concerns and remedies. Trends Genet. 2009;25:489-94.

66. Popejoy AB, Fullerton SM. Genomics is failing on diversity. Nature. 2016;538:161-4. 